\section{META-ANALYSIS OF CLINICAL TRIALS COMPARING TRIPLE VERSUS DUAL ANTITHROMBOTIC THERAPY IN PATIENTS WITH ATRIAL FIBRILLATION AND ACUTE CORONARY SYNDROMES OR CORONARY ARTERY DISEASE REQUIRING PERCUTANEOUS INTERVENTION}

Omar Assaf, Sabeeh Shams, Aysha Basir, Tawfiq Choudhury, Kenneth Wong. Blackpool Victoria Hospital, Blackpool, UK

\subsection{6/heartjnl-2021-BCS.41}

Aim To determine the optimum antithrombotic therapy for patients with atrial fibrillation (AF) and coronary artery disease (CAD) requiring percutaneous coronary intervention (PCI) and/or acute coronary syndrome (ACS) regarding the incidence of myocardial infarction or stent thrombosis. Previous meta-analyses have yielded conflicting conclusions. We have therefore performed a comprehensive literature search and performed a meta-analysis of all published RCTs to test the hypothesis that TAT significantly reduces the risk of stent thrombosis (ST) and Myocardial Infarction (MI).

Methods A literature search was done for RCTs on the efficacy of antithrombotic therapy in patients with AF and ACS or PCI.

Results A total of 11,542 patients were included in the 5 RCTs , comparing TAT vs DAT. Triple therapy did not lead to significant reduction in risk of Myocardial Infarction (MI) [risk ratio (RR) $0.85,95 \% \mathrm{CI} 0.69-1.04, \mathrm{P}=0.12$ ], [figure 1] or Stent Thrombosis (RR 0.76, 95\% CI 0.51-1.12 $\mathrm{P}=0.16)$. On the other hand, the risk of major bleeding or clinically significant bleeding is significantly lower with DAT [figure 2].

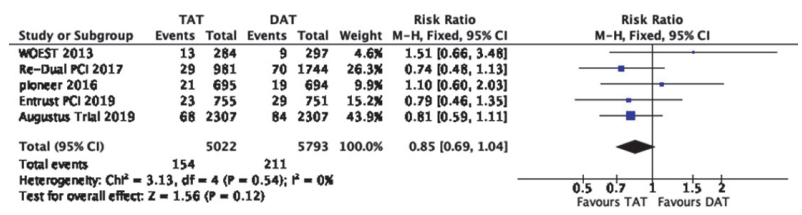

Abstract 41 Figure 1 Myocardial Infarction Risk is not significantly higher with DAT in any of the 5 RCTs or the meta-ana1vss

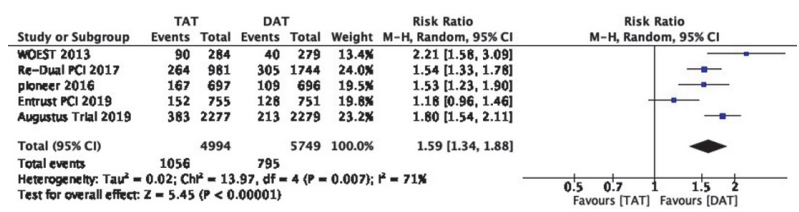

Abstract 41 Figure 2 Major Bleeding or clinically significant bleeding is significantly lower with DAT

Conclusion In conclusion, DAT comprising of warfarin or DOAC and P2Y12 inhibitor reduced risk of major or clinically significant bleeding without increasing the risk of ST or recurrent MI as compared to TAT. The results encourage the use of DAT rather than TAT in patients with ACS and/or patients requiring $\mathrm{PCI}$ with coexistent $\mathrm{AF}$ who require combined antiplatelet and anticoagulant therapy.

Conflict of Interest None

\section{CLINICAL SIGNIFICANCE OF EARLY ECHOCARDIOGRAPHY AFTER OUT-OF-HOSPITAL CARDIAC ARREST ON ARRIVAL TO A HEART ATTACK CENTRE}

Ritesh Kanyal, Dhruv Sarma, Nilesh Pareek, Rafal Dworakowski, Narbeh Melikian, Ian Webb, Ajay Shah, Philip MacCarthy, Jonathan Byrne. King's College Hospital, London, UK

\subsection{6/heartjnl-2021-BCS.42}

ArrestRitesh Kanyal, Dhruv Sarma, Nilesh Pareek, Rafal Dworakowski, Narbeh Melikian, Ian Webb, Ajay Shah, Philip MacCarthy, Jonathan Byrne.

Background Left ventricular systolic dysfunction (LVSD) is common after out of hospital cardiac arrest (OOHCA) and can manifest as global or regional change. PurposeWe evaluated the extent of global and regional LVSD and its association with coronary artery disease (CAD) and outcome in those undergoing coronary angiography after OOHCA.

Methods 619 patients with OOHCA were admitted at our centre between 1st May 2012 and 31st December 2017. After excluding 237 for having a non-cardiac aetiology/prior neurological disability, so 398 patients were included. Rates of cardiogenic shock and extent of CAD, as classified by the SYNTAX score were measured. The primary endpoint was 12 month mortality. Patients with incomplete data were excluded from the analysis.

Results Two hundred and sixty-six patients (median age 62 [53-71] 76.3\% male) underwent both trans-thoracic wchocardiography and coronary angiography on arrival and were included in the final analysis. $81.6 \%$ had ventricular fibrillation, $83.5 \%$ were witnessed and $51.9 \%$ occurred at residence. Ninety-six patients (36\%) had significant LVSD (Left Ventricular Ejection Fraction [LVEF] <40\%) and 139 (52.2\%) patients had regional wall motion abnormalities (RWMAs) on arrival. Patients were classified into 4 groups (Group A: LVEF $<40 \%$ / Global, Group B: LVEF $<40 \% /$ RWMA, Group C: LVEF $\geq 40 \%$ / Global and Group D: $L V E F \geq 40 \% / R W M A)$ with frequencies of $10.9 \%, 25,2 \%, 41.4 \%$ and 22.6\%). Patients in Group D had the shortest low-flow times and lowest rates of epinephrine administration, with most favourable metabolic status on arrival, based on lactate and creatinine values. In Groups B and D (RWMAs), patients were significantly more likely to

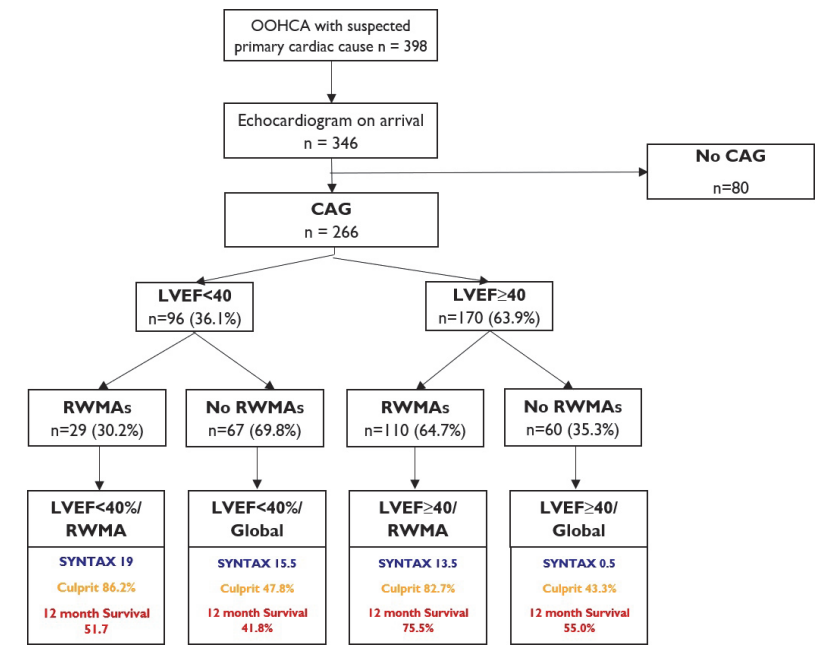

Abstract 42 Figure 1 